PROCEEDINGS OF THE

AMERICAN MATHEMATICAL SOCIETY

Volume 139, Number 1, January 2011, Pages 63-78

S 0002-9939(2010)10500-2

Article electronically published on July 19, 2010

\title{
PROOF OF THE ALDER-ANDREWS CONJECTURE
}

\author{
CLAUDIA ALFES, MARIE JAMESON, AND ROBERT J. LEMKE OLIVER
}

(Communicated by Kathrin Bringmann)

\begin{abstract}
Motivated by classical identities of Euler, Schur, and Rogers and Ramanujan, Alder investigated $q_{d}(n)$ and $Q_{d}(n)$, the number of partitions of $n$ into $d$-distinct parts and into parts which are $\pm 1(\bmod d+3)$, respectively. He conjectured that $q_{d}(n) \geq Q_{d}(n)$. Andrews and Yee proved the conjecture for $d=2^{s}-1$ and also for $d \geq 32$. We complete the proof of Andrews's refinement of Alder's conjecture by determining effective asymptotic estimates for these partition functions (correcting and refining earlier work of Meinardus), thereby reducing the conjecture to a finite computation.
\end{abstract}

\section{Introduction AND STATEMENT OF RESUlts}

A famous identity of Euler states that the number of partitions into odd parts equals the number of partitions into distinct parts, and the first Rogers-Ramanujan identity tells us that the number of partitions into parts which are $\pm 1(\bmod 5)$ equals the number of partitions into parts which are 2-distinct (a $d$-distinct partition is one where the difference between any two parts is at least $d$ ). Another related identity is a theorem of Schur which states that the partitions of $n$ into parts which are $\pm 1(\bmod 6)$ are in bijection with the partitions of $n$ into 3 -distinct parts where no consecutive multiples of 3 appear. In 1956, these three facts encouraged H. L. Alder to consider the partition functions $q_{d}(n):=p(n \mid d$-distinct parts) and $Q_{d}(n):=p(n \mid \operatorname{parts} \pm 1(\bmod d+3))$.

Conjecture (Alder). If $\Delta_{d}(n)=q_{d}(n)-Q_{d}(n)$, then, for any $d, n \geq 1$, we have that $\Delta_{d}(n) \geq 0$.

By the above discussion, the conjecture is true for $d \leq 3$, and the inequality can be replaced by an equality for $d=1$ and 2. Large tables of values seem to suggest, however, that $q_{d}(n)$ and $Q_{d}(n)$ are rarely equal. Andrews [1] refined Alder's conjecture (see [3] for more information on this conjecture):

Conjecture (Alder-Andrews). For $4 \leq d \leq 7$ and $n \geq 2 d+9$, or $d \geq 8$ and $n \geq d+6, \Delta_{d}(n)>0$.

Remark 1.1. For any given $d$, there are only finitely many $n$ not covered by the Alder-Andrews conjecture, and a simple argument shows that $\Delta_{d}(n) \geq 0$ for these $n$.

Received by the editors March 10, 2010

2010 Mathematics Subject Classification. Primary 11P82, 11P84.

(C)2010 American Mathematical Society Reverts to public domain 28 years from publication 
In essence, Alder's conjecture asks us to relate the coefficients of

$$
\sum_{n=0}^{\infty} Q_{d}(n) q^{n}=\prod_{n=1}^{\infty} \frac{1}{\left(1-q^{n(d+3)-(d+2)}\right)\left(1-q^{n(d+3)-1}\right)}
$$

and

$$
\sum_{n=0}^{\infty} q_{d}(n) q^{n}=\sum_{n=0}^{\infty} \frac{q^{d\left(\begin{array}{c}
n \\
2
\end{array}\right)+n}}{(1-q)\left(1-q^{2}\right) \cdots\left(1-q^{n}\right)} .
$$

Although the first generating function is essentially a weight 0 modular form, the second is generally not modular (except in the cases $d=1$ and 2). This is the root of the difficulty in proving Alder's conjecture, since the task is to relate the Fourier coefficients of two functions which have different analytic properties.

However, there have been several significant advances toward proving Alder's conjecture. Using combinatorial methods, Andrews [1] proved that Alder's conjecture holds for all values of $d$ which are of the form $2^{s}-1, s \geq 4$. In addition, Yee (9], 10]) proved that the conjecture holds for $d=7$ and for all $d \geq 32$. These results are of great importance because they resolve the conjecture except for $4 \leq d \leq 30, d \neq 7,15$.

In addition, Andrews [1] deduced that $\lim _{n \rightarrow \infty} \Delta_{d}(n)=+\infty$ using powerful results of Meinardus ([6], [7]) which give asymptotic expressions for the coefficients $q_{d}(n)$ and $Q_{d}(n)$. Unfortunately, a mistake in [7] implies that one must argue further to establish this limit. We correct the proof of Meinardus's main theorem (see the discussion after (3.11)) and show that the statement of the theorem remains unchanged. We first prove the following result, which can be made explicit:

Theorem 1.2. Let $d \geq 4$ and let $\alpha \in[0,1]$ be the root of $\alpha^{d}+\alpha-1=0$. If $A:=\frac{d}{2} \log ^{2} \alpha+\sum_{r=1}^{\infty} \frac{\alpha^{r d}}{r^{2}}$, then for every positive integer $n$ we have

$$
\Delta_{d}(n)=\frac{A^{1 / 4}}{2 \sqrt{\pi \alpha^{d-1}\left(d \alpha^{d-1}+1\right)}} n^{-3 / 4} \exp (2 \sqrt{n A})+\mathcal{E}_{d}(n),
$$

where $\mathcal{E}_{d}(n)=O\left(n^{-\frac{5}{6}} \exp (2 \sqrt{n A})\right)$.

Remark 1.3. The main term of $\Delta_{d}(n)$ is the same as the main term for $q_{d}(n)$ (cf. Theorem 3.1).

In the course of proving Theorem 1.2, we derive explicit approximations for $Q_{d}(n)$ and $q_{d}(n)$ (see Theorems 2.1 and 3.1, respectively). Using these results, we obtain the following:

Theorem 1.4. The Alder-Andrews Conjecture is true.

In order to prove Theorems 1.2 and 1.4, we consider $q_{d}(n)$ and $Q_{d}(n)$ independently and then compare the resulting effective estimates. Accordingly, in Section 2, we give explicit asymptotics for $Q_{d}(n)$, culminating in Theorem 2.1. Next, in Section 3. we laboriously make Meinardus's argument effective (and correct) in order to give an explicit asymptotic formula for $q_{d}(n)$ in Theorem 3.1 In Section 4 we use the results from Sections 2 and 3 to prove Theorems 1.2 and 1.4 . 


\section{Estimate of $Q_{d}(n)$ With EXPLicit ERROR Bound}

As before, let $Q_{d}(n)$ denote the number of partitions of $n$ whose parts are $\pm 1(\bmod d+3)$. From the work of Meinardus, we have that

$$
Q_{d}(n) \sim \frac{(3 d+9)^{-\frac{1}{4}}}{4 \sin \left(\frac{\pi}{d+3}\right)} n^{-\frac{3}{4}} \exp \left(n^{\frac{1}{2}} \frac{2 \pi}{\sqrt{3(d+3)}}\right) .
$$

In this formula, only the order of the error is known. We will bound the error explicitly, following closely the method of Meinardus [6] as it is presented by Andrews in Chapter 6 of [2]. This allows us to prove the following theorem:

Theorem 2.1. If $d \geq 4$ and $n$ is a positive integer, then

$$
Q_{d}(n)=\frac{(3 d+9)^{-\frac{1}{4}}}{4 \sin \left(\frac{\pi}{d+3}\right)} n^{-\frac{3}{4}} \exp \left(n^{\frac{1}{2}} \frac{2 \pi}{\sqrt{3(d+3)}}\right)+R(n),
$$

where $R(n)$ is an explicitly bounded function (see (2.10) at the end of this section).

Remark 2.2. An exact formula for $Q_{d}(n)$ is known from the work of Subrahmanyasastri [8]. In addition, by using Maass-Poincaré series, Bringmann and Ono [5] obtained exact formulas in a much more general setting. However, we do not employ these results since the formulas are extremely complicated, and Theorems 1.2 and 1.4 do not require this level of precision.

2.1. Preliminary facts. Consider the generating function $f$ associated to $Q_{d}(n)$,

$$
f(\tau):=\prod_{n \equiv \pm 1(d+3)}\left(1-q^{n}\right)^{-1}=1+\sum_{n=1}^{\infty} Q_{d}(n) q^{n},
$$

where $q=e^{-\tau}$ and $\Re(\tau)>0$. Let $\tau=y+2 \pi i x$. We can then obtain a formula for $Q_{d}(n)$ by integrating $f(\tau)$ against $e^{n \tau}$. Consequently, we require an approximation of $f(\tau)$ so that we may make use of this integral formula. To do this, we need an additional function,

$$
g(\tau):=\sum_{n \equiv \pm 1(d+3)} q^{n} .
$$

Lemma 2.3. If $\arg \tau>\frac{\pi}{4}$ and $|x| \leq \frac{1}{2}$, then $\Re(g(\tau))-g(y) \leq-c_{2} y^{-1}$, where $c_{2}$ is an explicitly given constant depending only on $d$.

Proof. We consider the expression $-y(\Re(g(\tau))-g(y))$. Expanding, we find that $-y(\Re(g(\tau))-g(y))=S_{1}+S_{2}+S_{3}$, where

$$
\begin{gathered}
S_{1}:=\frac{(1-\cos (2 \pi x))\left(e^{(3 d+8) y}-e^{(2 d+5) y}-e^{(d+4) y}+e^{y}\right)}{\left(\frac{e^{(d+3) y}-1}{y}\right)\left(e^{(2 d+6) y}-2 e^{(d+3) y} \cos (2 \pi(d+3) x)+1\right)}, \\
S_{2}:=\frac{(1-\cos (2 \pi(d+2) x))\left(e^{(2 d+7) y}-e^{(2 d+5) y}-e^{(d+4) y}+e^{(d+2) y}\right)}{\left(\frac{e^{(d+3) y}-1}{y}\right)\left(e^{(2 d+6) y}-2 e^{(d+3) y} \cos (2 \pi(d+3) x)+1\right)},
\end{gathered}
$$

and

$$
S_{3}:=\frac{(1-\cos (2 \pi(d+3) x))\left(2 e^{(2 d+5) y}+2 e^{(d+4) y}\right)}{\left(\frac{e^{(d+3) y}-1}{y}\right)\left(e^{(2 d+6) y}-2 e^{(d+3) y} \cos (2 \pi(d+3) x)+1\right)} .
$$

When $y=0$, we have $S_{1}=0, S_{2}=0$, and $S_{3}=\frac{2}{d+3}$. 
Since these functions are even in $x$, we may assume $x \geq 0$. Further, the condition $\arg \tau>\frac{\pi}{4}$ implies that $y<2 \pi x$. To find $c_{2}$, we note that each $S_{i} \geq 0$ and so it suffices to bound one away from 0 . We do this in three different cases.

Case 1. Suppose that $y \geq \frac{1}{2}$. Since $\frac{1}{2}>x>\frac{1}{2 \pi} y$, it follows that $1-\cos (2 \pi x)>$ $1-\cos \frac{1}{2}$ and that $S_{1}$ is bounded away from 0 . In particular,

$$
S_{1} \geq \frac{\pi\left(1-\cos \frac{1}{2}\right)\left(e^{\frac{3 d+8}{2}}-e^{\frac{2 d+5}{2}}-e^{\frac{d+4}{2}}+e^{\frac{1}{2}}\right)}{\left(e^{\pi(d+3)}-1\right)\left(e^{\pi(d+3)}+1\right)^{2}} .
$$

Case 2. Suppose that $y<\frac{1}{2}$ and $\left|x-\frac{k}{d+3}\right|<\frac{y}{d+3}$ for some positive integer $k$. Although less obvious than in Case $1, S_{1}$ will again be bounded away from 0 :

$$
S_{1} \geq \frac{\pi\left(1-\cos \frac{\pi}{d+3}\right)}{e^{\pi(d+3)}-1} \frac{e^{(3 d+8) y}-e^{(2 d+5) y}-e^{(d+4) y}+e^{y}}{\left(e^{(d+3) y}-1\right)^{2}+8 \pi^{2} y^{2} e^{(d+3) y}},
$$

and so

$$
S_{1} \geq \frac{2 \pi^{3}\left(1-\cos \frac{\pi}{d+3}\right)(d+2)(d+3)}{\left(e^{\pi(d+3)}-1\right)\left(\left(e^{(d+3) \pi}+1\right)^{2}+8 \pi^{4} e^{(d+3) \pi}\right)} .
$$

Case 3. Suppose that $y<\frac{1}{2}$ and $\frac{y}{d+3} \leq\left|x-\frac{k}{d+3}\right| \leq \frac{1}{2(d+3)}$ for some nonnegative integer $k$. This is permitted since every $x$ is covered as we vary $k$. It will be $S_{3}$ that is bounded away from 0 .

Let $u:=2 \pi(d+3)\left|x-\frac{k}{d+3}\right|$ and note that $0 \leq u \leq \pi, y \leq \frac{u}{2 \pi}$, and $\cos u=$ $\cos 2 \pi(d+3) x$. Now, we have that

$$
S_{3} \geq \frac{4 \pi}{e^{(d+3) \pi}-1} \frac{1-\cos u}{\left(e^{\frac{(d+3) u}{2 \pi}}-1\right)^{2}+2 e^{\frac{(d+3) u}{2 \pi}}(1-\cos u)},
$$

and a tedious analysis of the derivative of this function implies for $d \geq 4$ that

$$
S_{3} \geq \frac{8 \pi}{\left(e^{(d+3) \pi}-1\right)\left(\left(e^{\frac{d+3}{2}}-1\right)^{2}+4 e^{\frac{d+3}{2}}\right)} .
$$

Obviously, we may take $c_{2}$ to be the minimum of the bounds (2.1), (2.2), and (2.3).

Using Lemma 2.3, we now obtain an approximation for $f(\tau)$.

Lemma 2.4. If $|\arg \tau| \leq \frac{\pi}{4}$ and $|x| \leq \frac{1}{2}$, then

$$
f(\tau)=\exp \left(\frac{\pi^{2}}{3(d+3)} \tau^{-1}+\log \left(\frac{1}{2 \sin \frac{\pi}{d+3}}\right)+f_{2}(\tau)\right),
$$

where $f_{2}(\tau)=O\left(y^{\frac{1}{2}}\right)$ is an explicitly bounded function. Furthermore, if $y \leq y_{\max }$ is sufficiently small, $0<\delta<\frac{2}{3}, 0<\varepsilon_{1}<\frac{\delta}{2}, \beta:=\frac{3}{2}-\frac{\delta}{4}$, and $y^{\beta} \leq|x| \leq \frac{1}{2}$, then there is a constant $c_{3}$ depending on $d, \varepsilon_{1}$ and $\delta$ such that

$$
f(y+2 \pi i x) \leq \exp \left(\frac{\pi^{2}}{3(d+3)} y^{-1}-c_{3} y^{-\varepsilon_{1}}\right) .
$$


Remark 2.5. The discussion of the size of $y_{\max }$ will follow (2.4).

Proof. From page 91 of Andrews [2, we have that

$\log f(\tau)=\tau^{-1} \frac{\pi^{2}}{3(d+3)}+\log \left(\frac{1}{2 \sin \frac{\pi}{d+3}}\right)+\frac{1}{2 \pi i} \int_{-\frac{1}{2}-i \infty}^{-\frac{1}{2}+i \infty} \tau^{-s} \Gamma(s) \zeta(s+1) D(s) d s$,

where $D(s)$ is the Dirichlet series

$$
D(s):=\sum_{\substack{n \equiv \pm 1(d+3) \\ n \geq 0}} \frac{1}{n^{s}}
$$

which converges for $\Re(s)>1$. Writing

$$
D(s)=(d+3)^{-s}\left(\zeta\left(s, \frac{1}{d+3}\right)+\zeta\left(s, \frac{d+2}{d+3}\right)\right),
$$

where $\zeta(s, a)$ is the Hurwitz zeta function, we see that $D(s)$ can be analytically continued to the entire complex plane except for a pole of order 1 and residue $\frac{2}{d+3}$ at $s=1$ (see, for example, page 255 of Apostol's book [4]).

We bound the integral by noting that $|D(s)| \leq|\zeta(s)|$, obtaining

$$
\left|\frac{1}{2 \pi i} \int_{-\frac{1}{2}-i \infty}^{-\frac{1}{2}+i \infty} \tau^{-s} \Gamma(s) \zeta(s+1) D(s) d s\right| \leq \xi \sqrt{y},
$$

where

$$
\xi:=\frac{\sqrt{2}}{2 \pi} \int_{-\infty}^{\infty}\left|\zeta\left(\frac{1}{2}+i t\right) \zeta\left(-\frac{1}{2}+i t\right) \Gamma\left(-\frac{1}{2}+i t\right)\right| d t
$$

The first statement of the lemma follows.

Remark 2.6. Numerical estimates show that $\xi<.224$.

To prove the second statement, we again follow the method of Andrews [2]. We consider two cases: (1) $y^{\beta} \leq|x| \leq \frac{y}{2 \pi}$ and (2) $\frac{y}{2 \pi} \leq|x| \leq \frac{1}{2}$. In the first, we see that $|\arg \tau| \leq \frac{\pi}{4}$, so we apply the first statement of the lemma, getting

$$
\begin{aligned}
\log |f(y+2 \pi i x)| \leq & \frac{\pi^{2} y^{-1}}{3(d+3)}+\frac{\pi^{2} y^{-1}}{3(d+3)}\left(\left(1+4 \pi^{2} x^{2} y^{-2}\right)^{-\frac{1}{2}}-1\right) \\
& -\log \left(2 \sin \frac{\pi}{d+3}\right)+\xi \sqrt{y} \\
\leq & \frac{\pi^{2}}{3(d+3)} y^{-1}-c_{4} y^{-\frac{\delta}{2}}
\end{aligned}
$$

where

$$
c_{4}:=\frac{\pi^{4}}{3(d+3)}\left(2-\frac{3}{2} y_{\max }^{1-\frac{\delta}{2}}\right)-\log \left(\frac{1}{2 \sin \frac{\pi}{d+3}}\right) y_{\max }^{\frac{\delta}{2}}-\xi y_{\max }^{\frac{1+\delta}{2}} .
$$

In the second case, we have that

$$
\log |f(y+2 \pi i x)|=\log f(y)+\Re(g(\tau))-g(y),
$$

and using Lemma 2.3 , we obtain

$$
\log |f(y+2 \pi i x)| \leq \frac{\pi^{2}}{3(d+3)} y^{-1}-c_{5} y^{-1}
$$


where

$$
c_{5}:=c_{2}-y_{\max } \log \left(\frac{1}{2 \sin \frac{\pi}{d+3}}\right)-\xi y_{\max }^{\frac{3}{2}} .
$$

We let $c_{3}:=\min \left(c_{4}\left(y_{\max }\right)^{\varepsilon_{1}-\frac{\delta}{2}}, c_{5}\left(y_{\max }\right)^{\varepsilon_{1}-1}\right)$ and take $y_{\max }$ to be small enough so that $c_{3}>0$.

Remark 2.7. In the proof of Theorem 1.2, we need only bound $Q_{d}(n)$ since it is of lower order than $q_{d}(n)$. We shall ignore the restriction on $y_{\max }$ for convenience.

2.2. Proof of Theorem 2.1. From the Cauchy integral theorem, we have

$$
Q_{d}(n)=\frac{1}{2 \pi i} \int_{\tau_{0}}^{\tau_{0}+2 \pi i} f(\tau) \exp (n \tau) d \tau=\int_{-\frac{1}{2}}^{\frac{1}{2}} f(y+2 \pi i x) \exp (n y+2 n \pi i x) d x
$$

Applying the saddle point method, we take $y=n^{-\frac{1}{2}} \pi / \sqrt{3(d+3)}$ and we let $m:=$ $n y$ for notational simplicity. Assuming the notation in Lemma 2.4 for $n \geq 6$, we have $y_{\max } \leq\left(\frac{1}{2 \pi}\right)^{\frac{1}{\beta-1}}$, so that both cases in the proof of the second statement of Lemma 2.4 are nonvacuous. We have that

$$
Q_{d}(n)=e^{m} \int_{-y^{\beta}}^{y^{\beta}} f(y+2 \pi i x) \exp (2 \pi i n x) d x+e^{m} R_{1}
$$

where

$$
R_{1}:=\left(\int_{-\frac{1}{2}}^{-y^{\beta}}+\int_{y^{\beta}}^{\frac{1}{2}}\right) f(y+2 \pi i x) \exp (2 \pi i n x) d x
$$

By Lemma 2.4,

SO

$$
\left|R_{1}\right| \leq \exp \left[\frac{\pi^{2}}{3(d+3)}\left(\frac{m}{n}\right)^{-1}-c_{3}\left(\frac{m}{n}\right)^{-\varepsilon_{1}}\right]
$$

$$
\left|e^{m} R_{1}\right| \leq \exp \left(2 m-c_{3} m^{\varepsilon_{1}}\left(\frac{\pi^{2}}{3(d+3)}\right)^{-\varepsilon_{1}}\right)
$$

Using Lemma 2.4, write

$$
Q_{d}(n)=\exp \left(2 m-\log \left(2 \sin \frac{\pi}{d+3}\right)\right) \int_{-(m / n)^{\beta}}^{(m / n)^{\beta}} \exp \left(\varphi_{1}(x)\right) d x+\exp (m) R_{1}
$$

where

$$
\varphi_{1}(x):=m\left[\left(1+\frac{2 \pi i x n}{m}\right)^{-1}-1\right]+2 \pi i n x+g_{1}(x)
$$

and $\left|g_{1}(x)\right| \leq \xi \sqrt{\frac{\pi^{2}}{3 m(d+3)}}$.

After making the change of variables $2 \pi x=(m / n) \omega$, we obtain

$$
Q_{d}(n)=\exp \left(2 m+\log \frac{m}{n}+\log \left(\frac{1}{2 \sin \frac{\pi}{d+3}}\right)-\log (2 \pi)\right) I+\exp (m) R_{1}
$$

where

$$
I \quad:=\int_{-c_{10} m^{1-\beta}}^{c_{10} m^{1-\beta}} \exp \left(\varphi_{2}(\omega)\right) d \omega, \quad c_{10}:=2 \pi\left(\frac{\pi^{2}}{3(d+3)}\right)^{\beta-1}
$$


and

$$
\varphi_{2}(\omega):=m\left(\frac{1}{1+i \omega}-1+i \omega\right)+g_{1}(\omega) .
$$

We must now find an asymptotic expression for $I$. Write

$$
I=\int_{-c_{10} m^{1-\beta}}^{c_{10} m^{1-\beta}} \exp \left(-m \omega^{2}\right) d \omega+R_{2}
$$

where

with

$$
R_{2}:=\int_{-c_{10} m^{1-\beta}}^{c_{10} m^{1-\beta}} \exp \left(-m \omega^{2}\right)\left(\exp \left(\varphi_{3}(\omega)\right)-1\right) d \omega
$$

$$
\varphi_{3}(\omega):=m\left(\frac{1}{1+i \omega}-1+i \omega+\omega^{2}\right)+g_{1}(\omega) .
$$

Simplifying, we find that

$$
\left|\varphi_{3}(\omega)\right| \leq c_{10}^{3} m^{\frac{3 \delta-2}{4}}+\xi \sqrt{\frac{\pi^{2}}{3 m(d+3)}} .
$$

Substituting $m_{\min }=2^{\frac{2-\delta}{4}} \pi^{\frac{10-\delta}{4}}(3(d+3))^{-1}$ for $m$ in (2.7), it follows that

$$
\left|\varphi_{3}(\omega)\right| \leq \frac{2^{\frac{44+8 \delta-3 \delta^{2}}{16}} \pi^{\frac{76+8 \delta-3 \delta^{2}}{16}}}{3(d+3)}+\xi(2 \pi)^{\frac{\delta-2}{8}}=: \varphi_{3, \max }
$$

Thus, letting $c_{6}:=\frac{\exp \left(\varphi_{3, \max }\right)-1}{\varphi_{3, \max }}$, we have

$$
\left|\exp \left(\varphi_{3}(\omega)\right)-1\right| \leq m^{-\frac{1}{2}+\frac{3 \delta}{4}}\left(c_{6} c_{10}^{3}+\xi c_{6} m_{\min }^{-\frac{3 \delta}{4}} \sqrt{\frac{\pi^{2}}{3(d+3)}}\right)=: m^{-\frac{1}{2}+\frac{3 \delta}{4}} c_{7} .
$$

Hence, we conclude that $\left|R_{2}\right| \leq 2 c_{10} c_{7} m^{\delta-1}$.

Computing the integral in (2.6), we see that

$$
\int_{-c_{10} m^{1-\beta}}^{-c_{10} m^{1-\beta}} \exp \left(-m \omega^{2}\right) d \omega=\left(\frac{\pi}{m}\right)^{\frac{1}{2}}+g_{2}(m)
$$

where $\left|g_{2}(m)\right| \leq 2 m^{-\frac{1}{2}} \exp \left(-c_{10} m^{\frac{\delta}{4}}\right)$. Thus, we find that $I=\left(\frac{\pi}{m}\right)^{\frac{1}{2}}+g_{2}(m)+R_{2}$. Combining these results, we obtain the desired expression for $Q_{d}(n)$,

$$
Q_{d}(n)=\left(\frac{n^{-\frac{3}{4}}(3(d+3))^{-\frac{1}{4}}}{4 \sin \left(\frac{\pi}{d+3}\right)}\right) \exp \left(n^{\frac{1}{2}} \frac{2 \pi}{\sqrt{3(d+3)}}\right)+R(n),
$$

where

$$
\begin{aligned}
|R(n)| \leq n^{-\frac{1}{4}} & \left(\frac{\pi^{\frac{1}{2}(3(d+3))^{-\frac{3}{4}}}}{2 \sin \left(\frac{\pi}{d+3}\right)}\right) \exp \left(n^{\frac{1}{2}} \frac{2 \pi}{\sqrt{3(d+3)}}-n^{\frac{\delta}{8}} 2 \pi^{2-\frac{\delta}{4}}(3(d+3))^{-2+\frac{3 \delta}{8}}\right) \\
+ & n^{-1+\frac{\delta}{2}}\left(\frac{c_{7} \pi^{1+\frac{\delta}{2}}}{(3(d+3))^{2} \sin \left(\frac{\pi}{d+3}\right)}\right) \exp \left(n^{\frac{1}{2}} \frac{2 \pi}{\sqrt{3(d+3)}}\right) \\
& +\exp \left(n^{\frac{1}{2}} \frac{2 \pi}{\sqrt{3(d+3)}}-c_{3} n^{\frac{\varepsilon_{1}}{2}}\left(\frac{\pi^{2}}{3(d+3)}\right)^{-\frac{3 \varepsilon_{1}}{2}}\right)
\end{aligned}
$$




\section{Estimate of $q_{d}(n)$ WITH EXPLICIT ERROR BOUND}

Theorem 2 of [7] (with $k=m=1$ and $\ell=d$ ) gives

$$
q_{d}(n) \sim \frac{A^{1 / 4}}{2 \sqrt{\pi \alpha^{d-1}\left(d \alpha^{d-1}+1\right)}} n^{-3 / 4} \exp (2 \sqrt{n A}),
$$

where $\alpha$ and $A$ depend only on $d$ (see their definitions below in Theorem 3.1). We will bound the error explicitly, following closely the paper of Meinardus [7]. We make his calculations effective, and we obtain the following theorem.

Theorem 3.1. Let $\alpha$ be the unique real number in $[0,1]$ satisfying $\alpha^{d}+\alpha-1=0$, and let $A:=\frac{d}{2} \log ^{2} \alpha+\sum_{r=1}^{\infty} \frac{\alpha^{r d}}{r^{2}}$. If $n$ is a positive integer, then

$$
q_{d}(n)=\frac{A^{1 / 4}}{2 \sqrt{\pi \alpha^{d-1}\left(d \alpha^{d-1}+1\right)}} n^{-3 / 4} \exp (2 \sqrt{n A})+r_{d}(n),
$$

where $\left|r_{d}(n)\right|$ can be bounded explicitly (see the end of this section).

3.1. Preliminary facts. For fixed $d \geq 4$, we have the generating function

$$
f(z):=\sum_{n=0}^{\infty} q_{d}(n) e^{-n z}
$$

with $z=x+i y$. Hence, we obviously have that

$$
q_{d}(n)=\frac{1}{2 \pi} \int_{-\pi}^{\pi} f(z) e^{n z} d y
$$

Therefore to estimate $q_{d}(n)$ we require strong approximations for $f(z)$.

Lemma 3.2. Assuming the notation above, for $|y| \leq x^{1+\varepsilon}$ and $x<\beta$, where

$$
\beta:=\min \left(-\frac{\pi}{\log \rho} \xi, \frac{2 \alpha^{2-d}}{\pi d}, \frac{1}{2 d}+\rho\left(\frac{1}{2}-\frac{\pi^{2}}{24}\right)\right)^{\frac{1}{\varepsilon}},
$$

$\rho:=1-\alpha$, and $0<\xi<1$ is a constant, we have that

$$
f(z)=\left(\alpha^{d-1}\left(d \alpha^{d-1}+1\right)\right)^{-\frac{1}{2}} e^{\frac{A}{z}}\left(1+f_{\mathrm{err}}(z)\right),
$$

where $f_{\mathrm{err}}(z)=o(1)$ is an explicitly bounded function.

Lemma 3.3. Assuming the notation above, for $x<\beta$ and $x^{1+\varepsilon}<|y| \leq \pi$, we have that

$$
|f(x+i y)| \leq \sqrt{\frac{2 \pi}{d x}} e^{-\eta \rho x^{2 \varepsilon-1}}\left(1+f_{2}(\rho, x)\right) \exp \left(\frac{A}{x}+\frac{1-d}{2} \log \alpha+f_{1}(\rho, x)\right),
$$

where $f_{1}, f_{2}$ are functions given in Lemma 3.5, and $\eta$ is an explicitly given constant. Remark 3.4. Although $\varepsilon=\frac{11}{24}$ in [7, we will benefit by varying $\varepsilon$ in our work.

To prove Lemmas 3.2 and 3.3 , we follow 7 and write, by the Cauchy Integral Theorem,

$$
f(z)=\frac{1}{2 \pi i} \int_{\mathcal{C}} H(w, z) \Theta(w, z) \frac{d w}{w},
$$

where $\mathcal{C}$ is a circle of radius $\rho:=1-\alpha$ centered at the origin,

$$
H(w, z):=\prod_{n=1}^{\infty}\left(1-w e^{-n z}\right)^{-1}, \quad \text { and } \quad \Theta(w, z):=\sum_{n=-\infty}^{\infty} e^{-\frac{d}{2} n(n-1) z} w^{-n} .
$$


Therefore, we estimate $H(w, z)$ and $\Theta(w, z)$.

Lemma 3.5. Let $\rho=\alpha^{d}=1-\alpha$ and suppose that $w=\rho e^{i \varphi}$ with $-\pi \leq \varphi<\pi$. Then for $|y| \leq x^{1+\varepsilon}$ and $x<\beta$,

$$
H(w, z)=\exp \left(\frac{1}{z} \sum_{r=1}^{\infty} \frac{w^{r}}{r^{2}}+\frac{1}{2} \log (1-w)+f_{1}(w, z)\right)
$$

and

$$
\Theta(w, z)=\sqrt{\frac{2 \pi}{d z}} \exp \left(\frac{\log ^{2} w}{2 d z}-\frac{1}{2} \log w\right)\left(1+f_{2}(w, z)\right)
$$

where, as $x \rightarrow 0, f_{1}(w, z)=O\left(x^{\frac{1}{2}}\right)$ and $f_{2}(w, z)=O\left(x+e^{-\frac{c_{0}}{x}(\pi-|\varphi|)+c_{1} x^{\varepsilon-1}}\right)$ are explicitly bounded functions.

Proof. First, (3.4) and the inverse Mellin transform yield

$$
\log H(w, z)=\frac{1}{2 \pi i} \int_{2-i \infty}^{2+i \infty} z^{-s} \Gamma(s) \zeta(s) D(s+1, w) d s,
$$

where $\zeta(s)$ is the Riemann zeta function, $\Gamma(s)$ is the Gamma function, and $D(s, w):=$ $\sum_{r>1} \frac{w^{r}}{r^{s}}$, which is defined as a function of $s$ for all fixed $w$ with $|w|<1$.

Note that if $\theta_{0}:=\arctan x^{\varepsilon}$, then

$$
\left|z^{1 / 2-i t}\right| \leq|z|^{1 / 2} e^{\theta_{0}|t|} \leq\left(1+x^{2 \varepsilon}\right)^{\frac{1}{4}} x^{1 / 2} e^{\theta_{0}|t|}
$$

By changing the curve of integration and accounting for the poles at $s=0$ and 1 , we have

$$
\log H(w, z)=\frac{1}{z} \sum_{r \geq 1} \frac{w^{r}}{r^{2}}+\frac{\log (1-w)}{2}+f_{1}(w, z)
$$

where

$$
\begin{aligned}
\left|f_{1}(w, z)\right| & =\left|\frac{1}{2 \pi i} \int_{-\infty}^{\infty} z^{1 / 2-i t} \Gamma\left(-\frac{1}{2}+i t\right) \zeta\left(-\frac{1}{2}+i t\right) D\left(\frac{1}{2}+i t, w\right) d t\right| \\
& \leq\left(1+x^{2 \varepsilon}\right)^{\frac{1}{4}} 2^{-\frac{5}{2}} \pi^{-\frac{3}{2}} \zeta\left(\frac{3}{2}\right) \frac{\rho}{1-\rho} \frac{4}{\frac{\pi}{2}-\theta_{0}} x^{\frac{1}{2}}=: f_{1}(x) .
\end{aligned}
$$

This proves the first statement as $x^{2 \varepsilon}$ and $\theta_{0}=\arctan x^{\varepsilon}$ both tend toward 0 as $x \rightarrow 0$.

The transformation properties of theta functions give

$$
\Theta(w, z)=\sqrt{\frac{2 \pi}{d z}} e^{\frac{(\log w-d z / 2)^{2}}{2 d z}} \sum_{\mu=-\infty}^{\infty} e^{\frac{-2 \pi^{2} \mu^{2}}{d z}-\frac{2 \pi i \mu}{d z}(\log w-d z / 2)} .
$$


The argument on page 295 of [7] completes the proof of the lemma, with

$$
\begin{array}{r}
\left|f_{2}(w, z)\right| \leq e^{\frac{d|z|}{8}}\left[e^{\frac{d x \sqrt{1+x^{2 \varepsilon}}}{8}}-1+2 \frac{\exp \left(-\frac{4 \pi^{2}(1-\xi)}{d x\left(1+x^{2 \varepsilon}\right)}\right)}{1-\exp \left(-\frac{2 \pi^{2}(1-\xi)}{d x\left(1+x^{2 \varepsilon}\right)}\right)}\right] \\
\quad+2 \exp \left(\frac{2 \pi(|\varphi|-\pi)}{d x\left(1+x^{2 \varepsilon}\right)}-\frac{2 \pi \log \rho}{d} x^{\varepsilon-1}+\frac{d|z|}{8}\right) \\
=: f_{2}(\varphi, z)=: f_{2}^{0}(z)+f_{2}^{\varphi}(z) \exp \left(\frac{2 \pi|\varphi|}{d x\left(1+x^{2 \varepsilon}\right)}\right) .
\end{array}
$$

We now prove Lemmas 3.2 and 3.3 using Lemma 3.5

Proof of Lemma 3.2. Recall from (3.3) that

$$
f(z)=\frac{1}{2 \pi i} \int_{\mathcal{C}} H(w, z) \Theta(w, z) \frac{d w}{w} .
$$

Let $\varphi_{0}=x^{c}$ with $\frac{3}{8}<c<\frac{1}{2}$. Then

$$
f(z)=\frac{1}{2 \pi i} \int_{\rho e^{-i \varphi_{0}}}^{\rho e^{i \varphi_{0}}} H(w, z) \Theta(w, z) \frac{d w}{w}+\frac{1}{2 \pi i} \int_{\mathcal{B}} H(w, z) \Theta(w, z) \frac{d w}{w},
$$

where $\mathcal{B}$ is the circle $\mathcal{C}$ without the $\operatorname{arc} \rho e^{-i \varphi_{0}}$ to $\rho e^{i \varphi_{0}}$.

We first estimate the second integral in (3.10). We note the error of Meinardus [7] in the bound of $\Theta(w, z)$ provided between (25) and (26). From Lemma 3.5, we have that

$$
|\Theta(w, z)| \leq \sqrt{\frac{2 \pi}{d|z|}} \rho^{-\frac{1}{2}} \exp \left(\frac{x \log ^{2} \rho-\varphi^{2} x}{2 d\left(x^{2}+y^{2}\right)}+\frac{y \varphi \log \rho}{d\left(x^{2}+y^{2}\right)}\right)\left(1+\left|f_{2}(w, z)\right|\right) .
$$

The term $\frac{y \varphi \log \rho}{d\left(x^{2}+y^{2}\right)}$ does not appear in [7] and tends to infinity if $y \varphi<0$. This term arises from the main term of $\Theta(w, z)$, so its contribution cannot be ignored. Furthermore, it is $O\left(x^{\varepsilon-1}\right)$ and hence cannot be combined into the negative $O\left(x^{2 c-1}\right)$ term arising from $\frac{\varphi^{2} x}{2 d\left(x^{2}+y^{2}\right)}$. However, the bound Meinardus claims on the product $|H(w, z) \Theta(w, z)|$ is correct. To see this, we need more than the bound $|H(w, z)| \leq$ $H(\rho, x)$ that was originally thought to be sufficient.

From Lemma 3.5, we have that

$$
|H(w, z)| \leq \exp \left(\left|f_{1}(w, z)\right|\right)(1+\rho)^{\frac{1}{2}} \exp \left(\Re\left(\frac{1}{z} \sum_{r=1}^{\infty} \frac{w^{r}}{r^{2}}\right)\right)
$$

and

$$
\begin{aligned}
\Re\left(\frac{1}{z} \sum_{r=1}^{\infty} \frac{w^{r}}{r^{2}}\right)= & \frac{x}{x^{2}+y^{2}} \sum_{r=1}^{\infty} \frac{\rho^{r} \cos (r \varphi)}{r^{2}}+\frac{y}{x^{2}+y^{2}} \sum_{r=1}^{\infty} \frac{\rho^{r} \sin (r \varphi)}{r^{2}} \\
= & \frac{x}{x^{2}+y^{2}} \sum_{r=1}^{\infty} \frac{\rho^{r}}{r^{2}}+\frac{x}{x^{2}+y^{2}} \sum_{r=1}^{\infty} \frac{\rho^{r}}{r^{2}}(\cos (r \varphi)-1)-\frac{y \varphi \log (1-\rho)}{x^{2}+y^{2}} \\
& +\frac{y}{x^{2}+y^{2}} \sum_{r=1}^{\infty} \frac{\rho^{r}}{r^{2}}(\sin (r \varphi)-r \varphi) .
\end{aligned}
$$


Since $\rho=\alpha^{d}$ and $1-\rho=\alpha$, combining this with (3.12) and (3.11), we see that

$$
\begin{aligned}
|H(w, z) \Theta(w, z)| \leq & \sqrt{\frac{2 \pi}{d|z|}}\left(\frac{1+\rho}{\rho}\right)^{\frac{1}{2}} \exp \left(\left|f_{1}(w, z)\right|+\frac{A x}{x^{2}+y^{2}}-\frac{\varphi^{2} x}{x^{2}+y^{2}}\right. \\
& \left.\cdot\left[\frac{1}{2 d}+\frac{1}{\varphi^{2}} \sum_{r=1}^{\infty} \frac{\rho^{r}}{r^{2}}(1-\cos (r \varphi))-\frac{y}{\varphi^{2} x} \sum_{r=1}^{\infty} \frac{\rho^{r}}{r^{2}}(\sin (r \varphi)-r \varphi)\right]\right) \\
& \cdot\left(1+\left|f_{2}(w, z)\right|\right) .
\end{aligned}
$$

Hence, as $x \rightarrow 0$ we recover Meinardus's bound on $|H(w, z) \Theta(w, z)|$.

Using the notation of Lemma 3.5

$$
\begin{gathered}
\left|\int_{\mathcal{B}} H(w, z) \Theta(w, z) \frac{d w}{w}\right| \leq \sqrt{\frac{2 \pi}{d|z|}}\left(\frac{1+\rho}{\rho}\right)^{\frac{1}{2}} e^{f_{1}(x)+\frac{A x}{x^{2}+y^{2}}}\left[\left(1+f_{2}^{0}(z)\right) \int_{\mathcal{B}} e^{-\psi(\varphi, z)} d \varphi\right. \\
\left.+f_{2}^{\varphi}(z) \int_{\mathcal{B}} \exp \left(-\psi(\varphi, z)+\frac{2 \pi|\varphi|}{d x\left(1+x^{2 \varepsilon}\right)}\right) d \varphi\right]
\end{gathered}
$$

where

$\psi(\varphi, z):=\frac{\varphi^{2} x}{2 d\left(x^{2}+y^{2}\right)}+\frac{x}{x^{2}+y^{2}} \sum_{r=1}^{\infty} \frac{\rho^{r}}{r^{2}}(1-\cos (r \varphi))-\frac{y}{x^{2}+y^{2}} \sum_{r=1}^{\infty} \frac{\rho^{r}}{r^{2}}(\sin (r \varphi)-r \varphi)$.

We evaluate the two integrals of (3.13) separately.

For the integral $\int_{\mathcal{B}} \exp (-\psi(\varphi, z)) d \varphi$, we consider two parts based on the sign of $y \varphi$. We assume that $y>0$. When $\varphi>0, \sin (r \varphi)-r \varphi<0$ for all $r$, and so $\psi(\varphi, z)>0$. Then

$$
\begin{aligned}
\int_{\varphi_{0}}^{\pi} \exp (-\psi(\varphi, z)) d \varphi \leq & \frac{1}{\psi_{\varphi}\left(\varphi_{0}, z\right)}\left[\exp \left(-\psi\left(\varphi_{0}, z\right)\right)-\exp \left(-\psi\left(\nu \varphi_{0}, z\right)\right)\right] \\
& +\frac{1}{\psi_{\varphi}\left(\nu \varphi_{0}, z\right)}\left[\exp \left(-\psi\left(\nu \varphi_{0}, z\right)\right)-\exp (-\psi(\pi, z))\right]
\end{aligned}
$$

where $\nu>1$ is a constant.

When $\varphi<0$, we note that $\sin (r \varphi)-r \varphi>0$, and so

$$
\begin{aligned}
\psi(\varphi, z) & \geq \frac{\varphi^{2} x}{2 d\left(x^{2}+y^{2}\right)}+\frac{x}{x^{2}+y^{2}} \sum_{r=1}^{\infty} \frac{\rho^{r}}{r^{2}}(1-\cos (r \varphi))-\frac{x^{1+\varepsilon}}{x^{2}+y^{2}} \sum_{r=1}^{\infty} \frac{\rho^{r}}{r^{2}}\left(\frac{r^{3} \varphi^{3}}{6}\right) \\
& =\frac{\varphi^{2} x}{2 d\left(x^{2}+y^{2}\right)}+\frac{x}{x^{2}+y^{2}} \sum_{r=1}^{\infty} \frac{\rho^{r}}{r^{2}}(1-\cos (r \varphi))+\frac{\varphi^{3} x^{1+\varepsilon} \alpha^{d-2}}{6\left(x^{2}+y^{2}\right)} \\
& =: \hat{\psi}(\varphi, z),
\end{aligned}
$$

whence, using that $\frac{\pi^{2}}{2} \alpha^{d-2} x^{\varepsilon} \leq \frac{\pi}{d}$,

$$
\begin{aligned}
\int_{\varphi_{0}}^{\pi} \exp (-\psi(-\varphi, z)) d \varphi \leq & \frac{1}{\hat{\psi}_{\varphi}\left(-\varphi_{0}, z\right)}\left[\exp \left(-\hat{\psi}\left(-\varphi_{0}, z\right)\right)-\exp \left(-\hat{\psi}\left(-\nu \varphi_{0}, z\right)\right)\right] \\
& +\frac{1}{\hat{\psi}_{\varphi}\left(-\nu \varphi_{0}, z\right)}\left[\exp \left(-\hat{\psi}\left(-\nu \varphi_{0}, z\right)\right)-\exp (-\hat{\psi}(-\pi, z))\right] .
\end{aligned}
$$


We now consider the second integral in (3.13). A weaker bound on $\psi(\varphi, z)$ suffices. In particular, we have $\psi(\varphi, z) \geq k \varphi^{2}$, where

$$
k:=\frac{x}{x^{2}+y^{2}}\left(\frac{1}{2 d}-\frac{\pi \alpha^{d-2}}{6}\left|\frac{y}{x}\right|+\rho\left(\frac{1}{2}-\frac{\pi^{2}}{24}\right)\right),
$$

which is positive since $x<\beta$.

Hence, we have that

$$
\begin{aligned}
\int_{\mathcal{B}} \exp \left(-\psi(\varphi, z)+\frac{2 \pi|\varphi|}{d x\left(1+x^{2 \varepsilon}\right)}\right) d \varphi \leq \frac{d x\left(1+x^{2 \varepsilon}\right)}{\pi-k d x\left(1+x^{2 \varepsilon}\right)} \\
\cdot\left[\exp \left(-k \pi^{2}+\frac{2 \pi^{2}}{d x\left(1+x^{2 \varepsilon}\right)}\right)-\exp \left(-k \varphi_{0}^{2}+\frac{2 \pi \varphi_{0}}{d x\left(1+x^{2 \varepsilon}\right)}\right)\right] .
\end{aligned}
$$

Using (3.15), (3.17), and (3.18) in (3.13) gives an explicit bound for the second integral of (3.10), say $E_{\mathcal{B}}(z)$.

Following page 297 of 7 , the first integral of (3.10) is given by

$$
I:=\frac{1}{2 \pi i} \int_{\rho e^{-i \varphi_{0}}}^{\rho e^{i \varphi_{0}}} H(w, z) \Theta(w, z) \frac{d w}{w}=\frac{\exp \left(\frac{A}{z}+\frac{1-d}{2} \log \alpha\right)}{\sqrt{2 \pi d z}}\left(I_{\text {main }}+I_{\text {error }}\right),
$$

where

$$
I_{\text {main }}:=\int_{-\varphi_{0}}^{\varphi_{0}} \exp \left(-\frac{\varphi^{2}}{2 d z}\left(d \alpha^{d-1}+1\right)\right) d \varphi
$$

and

$$
\begin{gathered}
I_{\text {error }}:=\int_{-\varphi_{0}}^{\varphi_{0}}\left(\exp \left(\log \left(\frac{1-\rho e^{i \varphi}}{1-\rho}\right)+f_{3}(w, z)+f_{1}(w, z)\right)\left(1+f_{2}(w, z)\right)-1\right) \\
\cdot \exp \left(-\frac{\varphi^{2}}{2 d z}\left(d \alpha^{d-1}+1\right)\right) d \varphi
\end{gathered}
$$

where $\left|f_{3}(w, z)\right| \leq \frac{\rho e}{6(1-\rho e)^{2}} \varphi^{3}$. Then we have

$$
\left|I_{\text {error }}\right| \leq 2 \varphi_{0}\left(\frac{1-\rho \cos \varphi_{0}}{1-\rho} \exp \left(f_{1}(x)+\frac{\rho e}{6(1-\rho e)^{2}} \varphi_{0}^{3}\right)\left(1+f_{2}\left(\varphi_{0}, z\right)\right)-1\right)
$$

and

$$
I_{\text {main }}=\sqrt{\frac{\pi z d}{d \alpha^{d-1}+1}}-2 \int_{\varphi_{0}}^{\infty} \exp \left(-\frac{\varphi^{2}}{2 d z}\left(d \alpha^{d-1}+1\right)\right) d \varphi .
$$

Hence, it follows that

$$
I=\frac{\alpha^{\frac{1-d}{2}}}{\sqrt{d \alpha^{d-1}+1}} \exp \left(\frac{A}{z}\right)+\hat{E}_{\varphi_{0}}(w, z)
$$


where

$$
\begin{aligned}
\left|\hat{E}_{\varphi_{0}}(w, z)\right| \leq \frac{\alpha^{\frac{d-1}{2}}}{\sqrt{2 \pi d|z|}} e^{\frac{A x}{x^{2}+y^{2}}}\left[\frac{(2 d|z|)^{\frac{1}{2}}}{\varphi_{0}\left(d \alpha^{d-1}+1\right)^{\frac{1}{2}}} \exp \left(-\frac{\varphi_{0}^{2} x\left(d \alpha^{d-1}+1\right)}{2 d\left(x^{2}+y^{2}\right)}\right)\right. \\
\left.\quad+2 \varphi_{0}\left(\frac{1-\rho \cos \varphi_{0}}{1-\rho} \exp \left(f_{1}(x)+\frac{\rho e}{6(1-\rho e)^{2}} \varphi_{0}^{3}\right)\left(1+f_{2}\left(\varphi_{0}, z\right)\right)-1\right)\right] \\
=: E_{\varphi_{0}}(z) .
\end{aligned}
$$

Hence, we finally see that

$$
\left|f_{\mathrm{err}}(z)\right| \leq\left(E_{\varphi_{0}}(z)+E_{\mathcal{B}}(z)\right)\left(\alpha^{d-1}\left(d \alpha^{d-1}+1\right)\right)^{\frac{1}{2}} \exp \left(\frac{-A x}{x^{2}+y^{2}}\right) \text {. }
$$

Proof of Lemma 3.3. In order to bound $f$ for $x^{1+\varepsilon}<|y| \leq \pi$, note that $|\Theta(w, z)| \leq$ $\Theta(\rho, x)$ by (3.4), which also yields that

$$
\log |H(w, z)|=\Re\{\log H(w, z)\} \leq \log H(\rho, x)+\Re\left\{w \sum_{n \geq 1} e^{-n z}\right\}-\rho \sum_{n \geq 1} e^{-n x} .
$$

On the other hand, we have that

$$
\begin{aligned}
\Re\left\{w \sum_{n \geq 1} e^{-n z}\right\}-\rho \sum_{n \geq 1} e^{-n x} \leq & -\rho x^{2 \varepsilon-1}\left(\beta^{1-2 \varepsilon} e^{-\beta}\right) \\
& \cdot\left(\frac{1}{1-e^{-\beta}}-\frac{1}{\sqrt{1-2 e^{-\beta} \cos \beta^{1+\varepsilon}+e^{-2 \beta}}}\right) .
\end{aligned}
$$

To see this, note that

$$
\Re\left\{w \sum_{n \geq 1} e^{-n z}\right\}-\rho \sum_{n \geq 1} e^{-n x} \leq-\rho e^{-x}\left(\frac{1}{1-e^{-x}}-\frac{1}{\sqrt{1-2 e^{-x} \cos x^{1+\varepsilon}+e^{-2 x}}}\right) .
$$

This then gives

$$
\begin{aligned}
& \frac{\Re\left\{w \sum_{n \geq 1} e^{-n z}\right\}-\rho \sum_{n \geq 1} e^{-n x}}{}-\rho x^{2 \varepsilon-1} \\
& \quad \geq x^{1-2 \varepsilon} e^{-x}\left(\frac{1}{1-e^{-x}}-\frac{1}{\sqrt{1-2 e^{-x} \cos x^{1+\varepsilon}+e^{-2 x}}}\right) \\
& \quad \geq \beta^{1-2 \varepsilon} e^{-\beta}\left(\frac{1}{1-e^{-\beta}}-\frac{1}{\sqrt{1-2 e^{-\beta} \cos \beta^{1+\varepsilon}+e^{-2 \beta}}}\right)=: \eta
\end{aligned}
$$

The statement of Lemma 3.3 now follows from (3.3) and Lemma 3.5 . 
3.2. Proof of Theorem 3.1. From (3.2), it follows that $q_{d}(n)=I_{1}+I_{2}$, where

$$
I_{1}:=\frac{1}{2 \pi} \int_{-x^{1+\varepsilon}}^{x^{1+\varepsilon}} f(z) e^{n z} d y \quad \text { and } \quad I_{2}:=\frac{1}{2 \pi}\left(\int_{-\pi}^{-x^{1+\varepsilon}}+\int_{x^{1+\varepsilon}}^{\pi}\right) f(z) e^{n z} d y .
$$

In this proof, we let $x=\sqrt{\frac{A}{n}}$. Following the idea on page 291 of [7], we split $I_{1}$ as

$$
I_{1}=\gamma e^{\frac{2 A}{x}} \int_{-x^{1+\varepsilon}}^{x^{1+\varepsilon}} e^{-\frac{y^{2} A}{x^{3}}} d y+E_{2}+E_{3}
$$

where

$$
\gamma:=\frac{1}{2 \pi \sqrt{\alpha^{d-1}\left(d \alpha^{d-1}+1\right)}}, \quad E_{2}:=\gamma e^{\frac{2 A}{x}} \int_{-x^{1+\varepsilon}}^{x^{1+\varepsilon}} e^{-\frac{y^{2} A}{x^{3}}}\left(e^{A\left(\frac{y^{4}+i x y^{3}}{x^{3}\left(x^{2}+y^{2}\right)}\right)}-1\right) d y,
$$

and

$$
E_{3}:=\gamma e^{\frac{2 A}{x}} \int_{-x^{1+\varepsilon}}^{x^{1+\varepsilon}} e^{\frac{-x y^{2}+i y^{3}}{x^{2}\left(x^{2}+y^{2}\right)}} f_{\mathrm{err}}(z) d y
$$

The first integral in (3.25) can be written as

$$
\gamma e^{\frac{2 A}{x}} \int_{-x^{1+\varepsilon}}^{x^{1+\varepsilon}} e^{-\frac{y^{2} A}{x^{3}}} d y=\gamma e^{\frac{2 A}{x}} \sqrt{\frac{\pi x^{3}}{A}}+E_{1}
$$

where

$$
\left|E_{1}\right| \leq \frac{\gamma}{A \sqrt{2}} x^{2-\varepsilon} e^{\frac{2 A}{x}-A x^{2 \varepsilon-1}} .
$$

For $E_{2}$, we further split the integral:

$$
\begin{aligned}
E_{2} \quad & =\gamma e^{\frac{2 A}{x}} \int_{|y| \leq x^{1+\varepsilon_{2}}} e^{-\frac{y^{2} A}{x^{3}}}\left(e^{A\left(\frac{y^{4}+i x y^{3}}{x^{3}\left(x^{2}+y^{2}\right)}\right)}-1\right) d y \\
& +\gamma e^{\frac{2 A}{x}} \int_{x^{1+\varepsilon_{2}} \leq|y| \leq x^{1+\varepsilon}} e^{-\frac{y^{2} A}{x^{3}}}\left(e^{A\left(\frac{y^{4}+i x y^{3}}{x^{3}\left(x^{2}+y^{2}\right)}\right)}-1\right) d y
\end{aligned}
$$

with $\varepsilon_{2}>\varepsilon, \varepsilon_{2}>\frac{1}{3}$. Then

$$
\left|\gamma e^{\frac{2 A}{x}} \int_{|y| \leq x^{1+\varepsilon_{2}}} e^{-\frac{y^{2} A}{x^{3}}}\left(e^{A\left(\frac{y^{4}+i x y^{3}}{x^{3}\left(x^{2}+y^{2}\right)}\right)}-1\right) d y\right| \leq \gamma e^{\frac{2 A}{x}}\left(e^{A x^{3 \varepsilon_{2}-1}}-1\right) \sqrt{\frac{\pi x^{3}}{A}}
$$

and

$$
\begin{aligned}
& \left|\gamma e^{\frac{2 A}{x}} \int_{x^{1+\varepsilon_{2}} \leq|y| \leq x^{1+\varepsilon}} e^{-\frac{y^{2} A}{x^{3}}}\left(e^{A\left(\frac{y^{4}+i x y^{3}}{x^{3}\left(x^{2}+y^{2}\right)}\right)}-1\right) d y\right| \\
& \quad \leq \gamma \exp \left(\frac{2 A}{x}-\frac{A x^{\varepsilon_{2}-2}}{1+x^{2 \varepsilon}}\right) x^{3}\left(1+x^{2 \varepsilon}\right)+\frac{\gamma x^{3}}{A} \exp \left(\frac{2 A}{x}-A x^{\varepsilon_{2}-2}\right) .
\end{aligned}
$$

Finally, for $E_{3}$, we have

$$
\left|E_{3}\right| \leq \gamma e^{\frac{2 A}{x}}\left|f_{\operatorname{err}}^{\max }\right|\left(\pi x^{3}\left(1+x^{2 \varepsilon}\right)\right)^{\frac{1}{2}} .
$$

To bound $I_{2}$, we apply Lemma 3.3 to find that

$$
\left|I_{2}\right| \leq \sqrt{\frac{2 \pi}{d x}} e^{-\eta \rho x^{2 \varepsilon-1}}\left(1+f_{2}(\rho, x)\right) \exp \left(n x+\frac{A}{x}+\frac{1-d}{2} \log \alpha+f_{1}(\rho, x)\right) .
$$


Finally, we obtain

$$
q_{d}(n)=\frac{A^{1 / 4}}{2 \sqrt{\pi \alpha^{d-1}\left(d \alpha^{d-1}+1\right)}} n^{-3 / 4} \exp (2 \sqrt{n A})+E_{1}+E_{2}+E_{3}+I_{2},
$$

where $\left|E_{1}+E_{2}+E_{3}+I_{2}\right|$ is bounded using the expressions in (3.27)-(3.31). The result follows with $\left|r_{d}(n)\right| \leq\left|E_{1}\right|+\left|E_{2}\right|+\left|E_{3}\right|+\left|I_{2}\right|$.

\section{Proof of Alder's conjecture}

Using Theorems 2.1 and 3.1, we are now able to prove our main results.

Proof of Theorem 1.2. Applying the results of Sections 2 and 3 , we have that

$$
\Delta_{d}(n)=q_{d}(n)-Q_{d}(n)=\frac{A^{1 / 4}}{2 \sqrt{\pi \alpha^{d-1}\left(d \alpha^{d-1}+1\right)}} n^{-3 / 4} \exp (2 \sqrt{n A})+\mathcal{E}_{d}(n),
$$

where $\mathcal{E}_{d}(n)=r_{d}(n)-Q_{d}(n)$. In the proof of Lemma 2.4, we relax the restriction on $y_{\text {max }}$. Thus, Theorem 2.1 implies that $Q_{d}(n)=O\left(\exp \left(\frac{2 \pi}{\sqrt{3 d+9}} n^{1 / 2}+c_{0} n^{\frac{1}{6}}\right)\right)$, where $c_{0}$ is some positive constant.

By Theorem 3.1] $\left|r_{d}(n)\right| \leq\left|E_{1}\right|+\left|E_{2}\right|+\left|E_{3}\right|+\left|I_{2}\right|$, and a careful examination of each of these terms shows that $E_{1}=O\left(n^{-\frac{5}{6}} e^{2 \sqrt{A n}}\right), E_{2}=O\left(n^{-\frac{3}{2} \varepsilon_{2}-\frac{1}{4}} e^{2 \sqrt{A n}}\right)$, $E_{3}=O\left(n^{-\frac{15}{16}} e^{2 \sqrt{A n}}\right)$, and $I_{2}=O\left(n^{\frac{1}{4}} e^{2 \sqrt{A n}-\eta \rho x^{2 \varepsilon-1}}\right)$. Hence, by choosing $\varepsilon_{2} \geq$ $\frac{7}{18}$, the result follows.

Proof of Theorem 1.4. The works of Yee (9, [10]) and Andrews [1] show that $\Delta_{d}(n)$ is nonnegative when $d \geq 31$ and can be easily modified to show that $\Delta_{d}(n)$ is positive when $n \geq d+6$. For each remaining $4 \leq d \leq 30$, we use Theorems 2.1 and 3.1 to compute the smallest $n$ such that our bounds imply that $\Delta_{d}(n)>0$. We denote this $n$ by $\Omega(d)$, and a $\mathrm{C}++$ program computed the values of $\Delta_{d}(n)$ for $n \leq \Omega(d)$, which then confirmed the remaining cases of the Alder-Andrews Conjecture. As an example, we find that when $d=30, \Omega(30) \leq 9.77 \cdot 10^{6}$. To get this, we take $\delta=10^{-10}$ and $\varepsilon_{1}=5 \cdot 10^{-11}$ in Theorem 2.1, and in Theorem 3.1, $\varepsilon=.16906$, $\varepsilon_{2}=.499999, \xi=.99, c=.375000001$, and $\nu=1$. Other values of $d$ are similar and all satisfy $\Omega(d) \leq \Omega(30)$.

\section{ACKNOWLEDGEMENTS}

The authors thank Ken Ono for advising us on this project during the student number theory seminar at UW-Madison. The authors would also like to thank Kathrin Bringmann and Kimmo Eriksson for their helpful comments.

\section{REFERENCES}

[1] G. E. Andrews. On a partition problem of H. L. Alder. Pacific J. Math., 36:279-284, 1971. MR0289445 (44:6635)

[2] G. E. Andrews. The theory of partitions. Addison-Wesley Publishing Co., Reading, Mass.London-Amsterdam, 1976. Encyclopedia of Mathematics and its Applications, Vol. 2. MR0557013 (58:27738)

[3] G. E. Andrews and K. Eriksson. Integer partitions. Cambridge University Press, Cambridge, 2004. MR2122332 (2006b:11125)

[4] T. M. Apostol. Introduction to analytic number theory. Undergraduate Texts in Mathematics. Springer-Verlag, New York, 1976. MR0434929(55:7892) 
[5] K. Bringmann and K. Ono. Coefficients of harmonic Maass forms, Proceedings of the 2008 University of Florida Conference on Partitions, $q$-series, and modular forms, accepted for publication.

[6] G. Meinardus. Asymptotische Aussagen über Partitionen. Math. Z., 59:388-398, 1954. $\operatorname{MR} 0062781(16: 17 \mathrm{e})$

[7] G. Meinardus. Über Partitionen mit Differenzenbedingungen. Math. Z., 61:289-302, 1954. MR0068570(16:905a)

[8] V. V. Subrahmanyasastri. Partitions with congruence conditions. J. Indian Math. Soc. (N.S.), 36:177-194 (1973), 1972. MR0325560 (48:3907)

[9] A. J. Yee. Partitions with difference conditions and Alder's conjecture. Proc. Natl. Acad. Sci. USA, 101(47):16417-16418 (electronic), 2004. MR2114815 (2005i:05010)

[10] A. J. Yee. Alder's conjecture. J. Reine Angew. Math., 616:67-88, 2008. MR.2369487 (2008m:11205)

Lehrstuhl A für Mathematik, RWTh Aachen, Templergraben 64, D-52062 Aachen, GERMANY

Current address: Fachbereich Mathematik, Technische Universität Darmstadt, Schlossegartenstrasse 7, D-64289 Darmstadt, Germany

E-mail address: claudia.alfes@matha.rwth-aachen.de

Department of Mathematics, University of Wisconsin, Madison, Wisconsin 53706

Current address: Department of Mathematics and Computer Science, Emory University, Atlanta, Georgia 30322

E-mail address: marie.jameson@gmail.com

Department of Mathematics, University of Wisconsin, Madison, Wisconsin 53706

Current address: Department of Mathematics and Computer Science, Emory University, Atlanta, Georgia 30322

E-mail address: lemkeoliver@gmail.com 